\title{
Myocardial blood flow reserve and absolute myocardial blood flow for the assessment of patients with coronary artery disease with or without microvascular dysfunction
}

\author{
Raffaele Giubbini, $M D,{ }^{a, b}$ Elisabetta Cerudelli, $M D,{ }^{a}$ and Luca Camoni, $M S c^{a}$ \\ a Imaging Department and Nuclear Medicine Unit, University and Spedali Civili of Brescia, \\ Brescia, Italy \\ b Nuclear Medicine Department, Brescia, Italy
}

Received Jun 16, 2020; accepted Jun 16, 2020

doi: $10.1007 / \mathrm{s} 12350-020-02297-9$

See related article, pp. 2992-3003

The introduction of SPECT with CZT technology (solid-state detectors by Cadmium Zinc Telluride compounds) allows the dynamic acquisition of SPECT after tracer infusion. The input function and myocardial uptake can be easily measured. Algorithms for analysis of absolute myocardial blood flow (MBF) and myocardial blood flow reserve ratio (MFR, stress/rest MBF ratio) are currently provided by the principal Cardiac CZT vendors.

Several studies have recently documented the feasibility of MBF/MFR measurements by CZT technology in comparison to different reference standards, including positron emission tomography (PET) with $\mathrm{N}^{13} \mathrm{H}_{3}$ or $\mathrm{H}_{2} \mathrm{O}^{15},{ }^{1-4}$ invasive coronary angiography, ${ }^{5-8}$ magnetic resonance imaging, ${ }^{9}$ flow fractional reserve, ${ }^{5,10}$ and semiquantitative analysis of MPI. ${ }^{11}$ Several of the previously cited papers were accompanied by editorial comments ${ }^{12-15}$ which emphasized critical methodological limitations such as most clinical studies have been single site and enrolled a limited number of patients and

\footnotetext{
Reprint requests: Raffaele Giubbini, MD, Nuclear Medicine Department, Piazza Spedali Civili, 1, Brescia, Italy; raffaele.giubbini@unibs.it

J Nucl Cardiol 2021;28:3007-9.

$1071-3581 / \$ 34.00$

Copyright (C) 2020 American Society of Nuclear Cardiology.
}

the methodology is not standardized and varied from center to center.

Standardization of protocols requires addressing several aspects including (a) choice of the radiotracers, (b) tracer injection method (bolus vs. pump), (c) injected activity, (d) acquisition protocols (dual day vs single day, stress-rest vs. rest/stress), (e) kinetic models, (f) processing algorithms, (g) use of attenuation correction, scatter correction, spillover correction, partial volume correction, and motion correction, and (h) imaging systems. ${ }^{16}$

The group of the University of Ottawa Heart Institute has recently proposed a methodologic approach ${ }^{17}$ that is the first step for a multicenter validation study (NCT03427749) aimed to evaluate diagnostic accuracy, intra- and inter-observer variabilities, and incremental prognostic value of SPECT MBF over semiquantitative approaches. This study will provide an answer to many of the critical issues outlined above.

In this issue of the Journal of Nuclear Cardiology, Bailly et al proposed evaluation of the impact of attenuation correction (AC) of CZT-SPECT measurement of MBF. They applied a fast imaging protocol with 6-min dynamic acquisition after both stress and rest followed by a 5-min acquisition for static MPI imaging. They concluded that AC does not significantly affect MFR measurements in both regional and global LV analyses.

However, reading carefully the well-detailed and written paper the reader could conclude that AC strongly affects measurements of absolute MBF both at rest and after vasodilation stress test. Furthermore, the absolute MBF was lower after stress and rest than those obtained by PET with $\mathrm{N}^{13} \mathrm{H}_{3}$, or $\mathrm{H}_{2} \mathrm{O}^{15}$, ${ }^{18,19}$ especially for $\mathrm{AC}$ studies (values which are incompatible with life!). 
This underestimation could be related to suboptimal tuning of the adopted kinetic model. The strength of the study of Bailly et al is that the MFR is a parameter that is less affected by technological and methodological issues, being a ratio between two measures and, therefore, partially independent of the absolute values at rest and during hyperemia. However, it is mandatory to raise the question whether the measurement of MFR alone is enough to characterize macro- and micro-vascular dysfunction and myocardial ischemia.

In the study by Johnson and Gould ${ }^{20}$ it was clearly shown that an integration of MBF, MFR, and ischemic thresholds is needed for the assessment of the ischemic burden of coronary artery disease (CAD) and microvascular dysfunction.

MFR or stress flow alone failed to reflect ischemic burden in some patients. The MFR could be normal despite a low absolute stress MBF if the resting MBF is low and vice versa. We recognize that the above conditions affect only a minority of CAD patients, but at the same time the absence of a reliable measure of MBF at rest and during hyperemia limits the possibility of a correct classification of these patients. Gupta et $\mathrm{al}^{21}$ in 4029 consecutive patients studied by rest/stress PETMPI showed that both maximal MBF and MFR were strong predictors of major cardiac events and that the integrated assessment of the two indexes identified unique phenotypes of patients with known or suspected CAD who are at different risk for events. At least two studies with gamma-emitting perfusion tracers by CZT or traditional Anger cameras ${ }^{3,22}$ have shown the superiority of reconstruction algorithms which included motion and attenuation correction. Measuring MFR by CZT cameras requires additional time, due to the need of early dynamic acquisition for both stress and rest studies. The additional time to acquire a low-dose CT of the cardiac region by a stand-alone equipment is limited to few minutes, which compensates for the time needed to perform prone imaging.

In conclusion, the availability of a low-cost noninvasive technology for a reliable measure of MFR and MBF with a potential widespread distribution could open a new era in the evaluation of coronary microvascular disease with or without obstructive or non-obstructive atherosclerosis and may have important prognostic and therapeutic implications. ${ }^{23}$ MFR is a cornerstone in this process but an additional, reliable measure of MBF at rest and during hyperemia would be advisable. The results of a multicenter trial will define and standardize the methodology for the best approach to the measure of MBF and MFR by CZT technology with $99 \mathrm{mTc}$-labeled perfusion tracers.

\section{Disclosures}

The authors declare that they have no conflict of interest.

\section{References}

1. Agostini D, Roule V, Nganoa C, Roth N, Baavour R, Parienti JJ, et al. First validation of myocardial flow reserve assessed by dynamic (99m)Tc-sestamibi CZT-SPECT camera: Head to head comparison with (15)O-water PET and fractional flow reserve in patients with suspected coronary artery disease. The WATERDAY study. Eur J Nucl Med Mol Imaging 2018;45:1079-90.

2. Nkoulou R, Fuchs TA, Pazhenkottil AP, Kuest SM, Ghadri JR, Stehli J, et al. Absolute myocardial bloodflow and flow reserve assessed by gated SPECT with cadmium-zinc-telluride detectors using ${ }^{99 \mathrm{~m}} \mathrm{Tc}$-Tetrofosmin: Head-to-Head comparison with ${ }^{13} \mathrm{~N}$ Ammonia PET. J Nucl Med 2016;57:1887-92.

3. Giubbini R, Bertoli M, Durmo R, Bonacina M, Peli A, Faggiano I, et al. Comparison between $\mathrm{N}^{13} \mathrm{NH}_{3}$-PET and ${ }^{99 \mathrm{~m}} \mathrm{Tc}$-TetrofosminCZT SPECT in the evaluation of absolute myocardial blood flow and flow reserve. J Nucl Cardiol 2019. https://doi.org/10.1007/ s12350-019-01939-X.

4. Otaki Y, Manabe O, Miller RJ, Manrique A, Nganoa C, Roth N, et al. Quantification of myocardial blood flow by CZT-SPECT with motion correction and comparison with ${ }^{15} \mathrm{O}$-water PET. J Nucl Cardiol 2019. https://doi.org/10.1007/s12350-019-01854-1.

5. Bouallègue FB, Roubille F, Lattuca B, Cung TT, Macia JC, Gervasoni $R$, et al. SPECT myocardial perfusion reserve in patients with multivessel coronary disease: Correlation with angiographic findings and invasive fractional flow reserve measurements. J Nucl Med 2015;56:1712-7. https://doi.org/10.2967/ jnumed.114.143164.

6. Miyagawa M, Nishiyama Y, Uetani T, Ogimoto A, Ikeda S, Ishimura $\mathrm{H}$, et al. Estimation of myocardial flow reserve utilizing an ultrafast cardiac SPECT: Comparison with coronary angiography, fractional flow reserve, and the SYNTAX score. Int J Cardiol 2017;1:347-53.

7. de Souza ACDA, Gonçalves BK, Tedeschi AL, Lima RS. Quantification of myocardial flow reserve using a gamma camera with solid-state cadmium-zinc-telluride detectors: Relation to angiographic coronary artery disease. J Nucl Cardiol 2019. https://doi. org/10.1007/s12350-019-01775-Z.

8. Acampa W, Assante R, Mannarino T, Zampella E, D’Antonio A, Buongiorno $\mathrm{P}$, et al. Low-dose dynamic myocardial perfusion imaging by CZT-SPECT in the identification of obstructive coronary artery disease. Eur J Nucl Med Mol Imaging 2020;47:1705-12. https://doi.org/10.1007/s00259-019-04644-6.

9. Fang YHD, Liu YC, Ho KC, Kuo FC, Yang CF, Yen TC, et al. Single-scan rest/stress imaging with ${ }^{99 \mathrm{~m}} \mathrm{Tc}$-Sestamibi and cadmium zinc telluride-based SPECT for hyperemic flow quantification: A feasibility study evaluated with cardiac magnetic resonance imaging. PLoS ONE 2017;12:e0183402.

10. Zavadovsky KV, Mochula AV, Boshchenko AA, Vrublevsky AV, Baev AE, Krylov AL, et al. Absolute myocardial blood flows derived by dynamic CZT scan vs invasive fractional flow reserve: Correlation and accuracy. J Nucl Cardiol 2019. https://doi.org/10. 1007/s12350-019-01678-z.

11. Nudi F, Biondi-Zoccai G, Nudi A, Neri G, Procaccini E, Schilllaci O. Comparative analysis between myocardial perfusion reserve and maximal ischemia score at single photon emission computed tomography with new-generation cadmium-zinc-telluride cameras. J Nucl Cardiol 2019. https://doi.org/10.1007/s12350-019-01764-2. 
12. Liga R, Gimelli A. MPI in the era of CZT cameras: Absolute numbers are still better than relative figures. J. Nucl. Cardiol 2019. https://doi.org/10.1007/s12350-019-01777-x.

13. Pelletier-Galarneau M, Ruddy TD. A big step towards clinical implementation of myocardial blood flow quantification with CZT SPECT. J Nucl Cardiol 2019. https://doi.org/10.1007/s12350-01901894-7.

14. Zampella E, Assante R, Gaudieri V, Nappi C, Acampa W, Cuocolo A. Myocardial perfusion reserve by using CZT: It's a long way to the top if you wanna standardize. J Nucl Cardiol 2019. h ttps://doi.org/10.1007/s12350-019-01817-6.

15. Bajaj NS, Bhambhvani P. SPECT-derived absolute myocardial perfusion measures: A step in the right direction. J Nucl Cardiol 2019. https://doi.org/10.1007/s12350-019-01972-w.

16. Zoccarato O, Lizio D, Savi A, Indovina L, Scabbio C, Leva L, et al. Comparative analysis of cadmium-zincum-telluride cameras dedicated to myocardial perfusion SPECT: A phantom study. J Nucl Cardiol 2016;23:885-93.

17. Wells RG, Marvin B. Measuring SPECT myocardial blood flow at the University of Ottawa Heart Institute. J Nucl Cardiol 2020. h ttps://doi.org/10.1007/s12350-020-02102-7.

18. Nagamachi S, Czernin J, Kim AS, et al. Reproducibility of measurements of regional resting and hyperemic myocardial blood flow assessed with PET. J Nucl Med 1996;37:1626-31.
19. Chareonthaitawee P, Kaufmann PA, Rimoldi O, Camici PG. Heterogeneity of resting and hyperemic myocardial blood flow in healthy humans. Cardiovasc Res 2001;50:151-61.

20. Johnson NP, Gould KL. Integrating noninvasive absolute flow, coronary flow reserve, and ischemic thresholds into a comprehensive map of physiological severity. JACC Cardiovasc Imaging 2012;5:430-40. https://doi.org/10.1016/j.jcmg.2011.12.014.

21. Gupta A, Taqueti VR, van de Hoef TP, et al. Integrated noninvasive physiological assessment of coronary circulatory function and impact on cardiovascular mortality in patients with stable coronary artery disease. Circulation 2017;136: 2325-36.

22. Wells RG, Marvin B, Poirier M, Renaud J, deKemp RA, Ruddy TD. Optimization of SPECT measurement of myocardial blood flow with corrections for attenuation, motion, and blood binding compared with PET. J Nucl Med 2017;58:2013-9. https://doi.org/ 10.2967/jnumed.117.191049.

23. Taqueti VR, Di Carli MF. Coronary microvascular disease pathogenic mechanisms and therapeutic options: JACC state-ofthe-art review. J Am Coll Cardiol 2018;72(21):2625-41.

Publisher's Note Springer Nature remains neutral with regard to jurisdictional claims in published maps and institutional affiliations. 